\title{
Successful removal of kinked J-guide wire under fluoroscopic guidance during central venous catheterization
}

\section{-A case report-}

\author{
Hyun-Seok Han ${ }^{2}$, Young-Tae Jeon ${ }^{1}$, Hyo-Seok Na ${ }^{1}$, Jin-Young Hwang ${ }^{1}$, Eun-Joo Choi ${ }^{1}$, and Mi-Hyun Kim ${ }^{1}$
}

Department of Anesthesiology and Pain Medicine, ${ }^{1}$ Seoul National University Bundang Hospital, Seongnam, ${ }^{2}$ Seoul National University College of Medicine, Seoul, Korea

Guidewire-associated complications that occur during the process of central venous catheterization include its kinking, looping, knotting and breakage. The removal of a looped or knotted guidewire is problematic because it can cause vessel damage, major hemorrhage, or embolization of a fractured guidewire. We report a case of guidewire kinking and its successful removal under fluoroscopic guidance. (Korean J Anesthesiol 2011; 60: 362-364)

Key Words: Central venous catheterization, Guide wire, Kinking.

Central venous catheters are frequently used with many patients for the delivery of medication, intravenous fluids, parenteral nutrition, hemodialysis, and the monitoring of hemodynamic variables. Despite its utility, the rate of mechanical complications, such as arterial puncture, pulmonary embolism, air embolism, nerve injury, hemothorax or pneumothorax, and dysrhythmia, is reported to be between 5 and $29 \%$ [1,2]. Central venous cannulation is commonly performed using the Seldinger technique, which mandates the use of a guidewire $[3,4]$. Complications associated with guidewire use include its kinking, looping, knotting and breakage $[5,6]$. We present a case of kinking of a guidewire during attempted central venous catheterization, and its successful removal under fluoroscopic guidance.

\section{Case Report}

A previously healthy 24-year-old, $67 \mathrm{~kg}, 164 \mathrm{~cm}$ woman, who had been diagnosed with olfactory groove meningioma, was scheduled to undergo craniotomy and tumor removal. Anesthesia was induced, with an effect site concentration of 4 $\mu \mathrm{g} / \mathrm{ml}$ propofol and $2 \mathrm{ng} / \mathrm{ml}$ remifentanil, by target controlled infusion (TCI); $50 \mathrm{mg}$ rocuronium was given to facilitate tracheal intubation.

While anesthesia was subsequently maintained by TCI of $3 \mu \mathrm{g} / \mathrm{ml}$ propofol and $2 \mathrm{ng} / \mathrm{ml}$ remifentanil, insertion of a central venous catheter through the right subclavian vein was attempted with two lumen indwelling catheter sets (ARROWguard Blue, Arrow International INC., USA). The

Received: July 30, 2010. Revised: 1st, October 6, 2010; 2nd, November 28, 2010. Accepted: December 15, 2010.

Corresponding author: Mi-Hyun Kim, M.D., Department of Anesthesiology and Pain Medicine, Seoul National University Bundang Hospital, 166, Gumi-ro, Bundang-gu, Seongnam 463-707, Korea. Tel: 82-31-787-2034, Fax: 82-31-787-4025, E-mail: 80274@snubh.org

(ㄷ) This is an open-access article distributed under the terms of the Creative Commons Attribution Non-Commercial License (http:// creativecommons.org/licenses/by-nc/3.0/), which permits unrestricted non-commercial use, distribution, and reproduction in any medium, provided the original work is properly cited. 
introducer needle was advanced at the infraclavicular site, $1 \mathrm{~cm}$ inferior to distal third of the clavicle towards the suprasternal notch, until free aspiration of blood was obtained. A J-shaped guide wire was passed through the venipuncture needle $20 \mathrm{~cm}$ beyond the needle tip.

Following removal of the needle, tissue dilator was threaded over the wire. Slight resistance was felt, so the dilator was advanced by further application of force. The catheter was put into the skin after removal of the dilator, but it did not advance any further than $7 \mathrm{~cm}$ because of resistance; second and third attempts were unsuccessful. Therefore, it was decided that the wire should be removed. The catheter was removed, but the guide wire could not be retracted, despite firm traction.

Fluoroscopy showed the guidewire to be kinked near the periclavicular area (Fig. 1). Following discussion with thoracic surgeon, it was decided that removal of the wire, along with the dilator, under fluoroscopic guidance should be attempted. Under fluoroscopic guidance, tissue dilator was inserted to straighten the wire, and the wire was removed along with the dilator. Because significant force was required to withdraw the wire, the inner filament of the wire broke (Fig. 2). The gap between the divided ends was bridged by portion of coiled wire-cover that surrounded the inner filament (Fig. 2).

Right internal jugular vein catheterization and surgery was performed uneventfully. A postoperative chest X-ray, taken in the surgical intensive care unit revealed no signs of hemothorax or pneumothorax.

\section{Discussion}

We present a case of kinking of a J-tipped guidewire during a

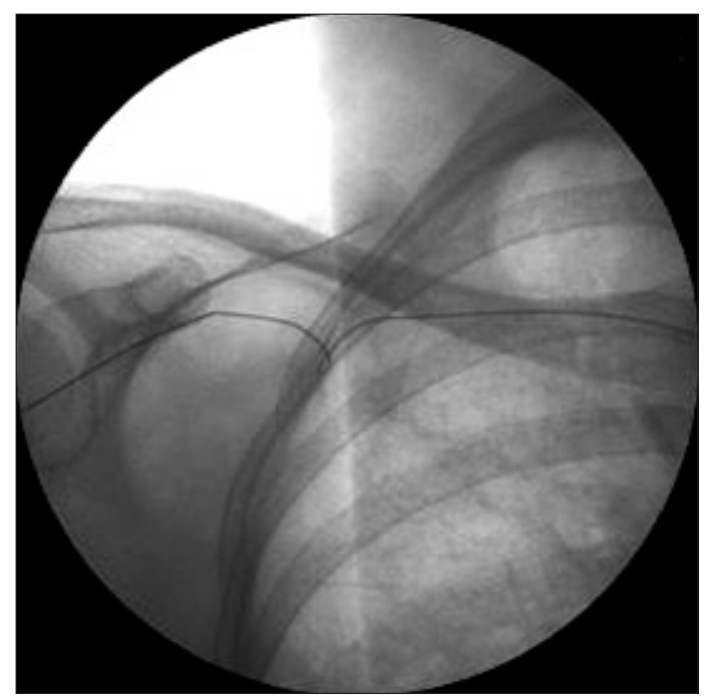

Fig. 1. Fluoroscopy shows kinking of the guidewire at the periclavicular area. subclavian vein cannulation attempt. A few cases of knotting, kinking, or entrapment of guidewires have been reported, and previous reports of this complication have performed a simple retraction without fluoroscopic guidance $[7,8]$. In our case, the kinked guidewire was removed successfully with fluoroscopic guidance.

To establish the etiology of kinking or knotting of guidewire, it is necessary to have an understanding of the structure and physical characteristics of the wires. Guidewire consists of a single inner filament and a surrounding coiled wire. The latter is designed as a helix to form a tunnel for the inner wire, and provides elastic properties. J-shaped curvature is achieved by flattening the round core to a thickness of $0.1 \mathrm{~mm}$ approximately $2.5 \mathrm{~cm}$ from the tip, which provides flexibility at the J-shaped tip. However, it becomes an area of structural weakness at the junction of the rounded and flattened segments, which can lead to potential breakage [9]. According to testing of guidewires, the welded seams between the inner core and the outer covering at both ends are supposed to withstand tension of at least 17 $\mathrm{N}$ ( $1.73 \mathrm{~kg}$ force) before they detach; whereas in its middle section, the complete guide wire should withstand a stress of approximately $290 \mathrm{~N}$ (29.57 kg force) [6]. Guidewires are flexible structures, which means they are likely to become kinked or knotted if any force is applied repeatedly or excessively $[6,10]$. Also, some maneuvers may damage the guide wire; for example, as occurred in this report, excessive pushing tension was required to insert the dilator. Furthermore, the dilator may have been forced to advance in a direction that diverged from the path of the wire, which led to kinking of the wire.

A kinked or knotted guide wire should be removed carefully, as it can induce vessel injury, hemorrhage or embolization of the fractured wire. In some publications, the guide wire was removed by retraction without confirming the condition of the guide wire with a radiologic device $[7,8]$. There is one other report in which a tissue dilator was used to tighten the knot and straighten the kinking wire, allowing it to be withdrawn along with the dilator such; as was performed in

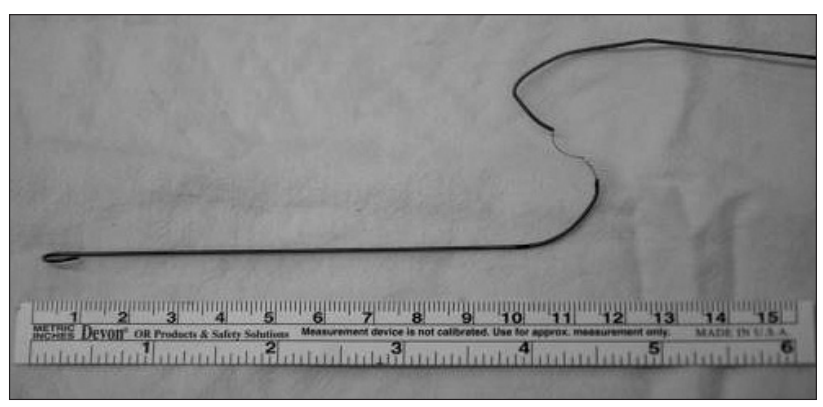

Fig. 2. The broken inner part of the guidewire, after its retrieval, is shown. 
our case [9]. However, such blind maneuvers may result in fatal consequences such as tissue injury, major hemorrhage, or embolization of fractured wire fragments $[11,12]$. In our case, to avoid these severe complications, careful retraction during removal of the guidewire was carried out under fluoroscopic guidance. We used dilator to straighten the wire and remove it. The dilator was used to tighten and reduce the knot to its smallest diameter. Because the circumference of the knot was the knotted wire could be removed with dilator; i.e. the dilator and guide wire can be withdrawn as a unit. Slight resistance was felt on attempting to remove the guidewire, and considerable force was required to remove the wire. As a consequence, the inner filament of the guidewire was broken. However, with the help of fluoroscopy, it was possible to prevent complete breakage of guidewire. The use of fluoroscopy can allow for early diagnosis of kinking or knotting of the guidewire, and realtime monitoring its structure during removal. For these reasons, fluoroscopy potentially promotes safer retrieval of guidewire.

When breakage or embolization of the guidewire occurs, a basket retrieval catheter or loopsnare catheter can be used for removal of the fragments under fluoroscopic guidance. These percutaneous retrieval procedures have been reported to have success rate of $92 \%$ [12]. Surgical intervention is only considered if all of these methods fail to remove the wire $[10,13]$.

Kinking, knotting and any further damage to the structure of the guidewires can only occur because of physical tension during their handling. Therefore, we suggest that guidewires should not be advanced by application of force if any resistance is detected. Fluoroscopic control is recommended for safer removal of knotted or kinked guidewires.

\section{References}

1. Eisen LA, Narasimhan M, Berger JS, Mayo PH, Rosen MJ, Schneider RF. Mechanical complications of central venous catheters. J Intensive Care Med 2006; 21: 40-6.

2. McGee DC, Gould MK. Preventing complications of central venous catheterization. N Engl J Med 2003; 348: 1123-33.

3. SELDINGER SI. Catheter replacement of the needle in percutaneous arteriography; a new technique. Acta radiol 1953; 39: 368-76.

4. Sternbach G. Sven Ivar Seldinger: catheter introduction on a flexible leader. J Emerg Med 1990; 8: 635-7.

5. Carpentier JP, Braz da Silva J, Choukroun G. Formation of a knot in a J spiral metallic guide: a complication of the Seldinger method. Cah Anesthesiol 1991; 39: 277-8.

6. Monaca E, Trojan S, Lynch J, Doehn M, Wappler F. Broken guide wire--a fault of design? Can J Anaesth 2005; 52: 801-4.

7. Kim SY, Jang YH. J-guide Wire Knotting during the Central Venous Catheterization. Korean J Anesthesiol 2003; 44: 725-8.

8. Kim HK, Choi JW, Lee JH, Lee AR. Knotting of Guidewire during internal jugular vein catheterization in an infant. Korean J Anesthesiol 2009; 57: 531-4.

9. Khan KZ, Graham D, Ermenyi A, Pillay WR. Case report: managing a knotted Seldinger wire in the subclavian vein during central venous cannulation. Can J Anaesth 2007; 54: 375-9.

10. Lee JJ, Kim JS, Jeong WS, Kim DY, Hwang SM, Lim SY. A complication of subclavian venous catheterization: extravascular kinking, knotting, and entrapment of the guidewire -A case report. Korean J Anesthesiol 2010; 58: 296-8.

11. Reddy SC, Kamath P, Talwar KK, Saxena A, Wasir HS. Guide wire outer coat shearing and embolisation: an unusual complication of pericardiocentesis. Int J Cardiol 1997; 60: 15-8.

12. Yang FS, Ohta I, Chiang HJ, Lin JC, Shih SL, Ma YC. Non-surgical retrieval of intravascular foreign body: experience of 12 cases. Eur J Radiol 1994; 18: 1-5.

13. Struck MF, Kaden I, Heiser A, Steen M. Cross-over endovascular retrieval of a lost guide wire from the subclavian vein. J Vasc Access 2008; 9: 304-6. 